\title{
Sex specific trajectories of central adiposity, lipid indices, and glucose level with incident hypertension: 12 years Follow-up in Tehran lipid and glucose study
}

\author{
Noushin Sadat Ahanchi', Seyed Saeed Tamehri Zadeh², Davood Khalili' ${ }^{1}, 3$, Fereidoun Azizi ${ }^{4}$ \\ and Farzad Hadaegh ${ }^{1^{*}}$ (D)
}

\begin{abstract}
Aims: To identify sex specific trajectories of waist circumference (WC), triglyceride (TG), total cholesterol (TC), high density lipoprotein cholesterol ( $\mathrm{HDL}-\mathrm{C}$ ) and fasting plasma glucose (FPG) during adulthood and examine their associations with incident hypertension.

Methods: The cohort consisted of 5030 participants (2051 males) with at least 2 repeated measurement during a median of 12 years follow up. We identified trajectory groups using latent class growth mixture model, their association with hypertension was examined using multivariate Cox-regression analysis.

Results: We found 997 cases of hypertension (483 male). For both exposures, three distinct trajectory groups were identified in both genders. For WC, in women: low-increasing, 82.4\%; high-stable, 13.4\%; high-increasing, 4.2\% and in men: stable, 94.6\%; low-increasing, 3.6\% and for high-increasing, 1.7\%. For TG, in women: stable, 91.3\%; decreasing, 5.9\%; inverse U-shape, 2.8\%; in men: stable, 89.7\%; inverse U- shape, 6.2\% and for decreasing, $4.1 \%$.

Regarding WC, high stable and high-increasing trajectories were associated with hypertension in the multivariate model [(hazard ratio $(\mathrm{HR})=1.66(95 \% \mathrm{Cl} 1.26-2.20)$ and $2.78(1.79-3.60)$, respectively]. Among men, this association was shown only for the low-increasing trajectory [2.76: 1.49-5.10]. For TG, among women decreasing and inverse U-shape trajectories were significantly associated with hypertension in the multivariate model [1.32:1.01-1.76] and [2.23:1.58-3.23, respectively].
\end{abstract}

We did not find any impact of increasing trajectories of FPG and HDL-C on incident hypertension. Considering TC, all individuals followed a stable trajectory.

Conclusion: WC dynamic changes in both gender and TG trajectory among women were significantly associated with incident hypertension.

Keywords: Waist circumference, Triglyceride, Trajectory, Hypertension

\footnotetext{
*Correspondence: fzhadaegh@endocrine.ac.ir

${ }^{1}$ Prevention of Metabolic Disorders Research Center, Research Institute for Endocrine Sciences, Shahid Beheshti University of Medical Sciences, No. 24, Parvaneh Street, Velenjak, Tehran, Iran

Full list of author information is available at the end of the article
}

\section{Introduction}

Based on many years of experience, hypertension significantly increases individuals' vulnerability to cardiovascular disease (CVD) and mortality. Despite tremendous advancements regarding hypertension prevention and treatment, itremainsa global concern, especially in the

c) The Author(s) 2021. This article is licensed under a Creative Commons Attribution 4.0 International License, which permits use, sharing, adaptation, distribution and reproduction in any medium or format, as long as you give appropriate credit to the original author(s) and the source, provide a link to the Creative Commons licence, and indicate if changes were made. The images or other third party material in this article are included in the article's Creative Commons licence, unless indicated otherwise in a credit line to the material. If material is not included in the article's Creative Commons licence and your intended use is not permitted by statutory regulation or exceeds the permitted use, you will need to obtain permission directly from the copyright holder. To view a copy of this licence, visit http://creativeco mmons.org/licenses/by/4.0/. The Creative Commons Public Domain Dedication waiver (http://creativecommons.org/publicdomain/ zero/1.0/) applies to the data made available in this article, unless otherwise stated in a credit line to the data. 
Middle East and North Africa (MENA) region [1, 2]. Apart from CVD, hypertension is a major factor in the development of end-stage renal disease, heart failure, and premature mortality [3]. Approximately $40 \%$ of the people of the Eastern Mediterranean region (EMR) suffer from hypertension, which is similar to the prevalence of global hypertension among adults who are older than 25 [4].

Lines of research have been performed so as to identify the possible risk factors implicated in hypertension development $[5,6]$. An updated systemic review has addressed the pivotal role of several factors including, but not limited to, age, sex, body mass index (BMI), waist circumference (WC), lipid abnormalities, smoking and diabetes in hypertension [6]. It is of importance to note that the majority of studies assessing the association between potential risk factors and incident hypertension have focused on a snapshot measurement of those and ignored the impact of their dynamic changes on incident hypertension.

Although the association between general adiposity and incident hypertension has been investigated in several research $[7,8]$, to the best of our knowledge, so far there is no information available on the effects of central adiposity, lipid indices including triglyceride (TG), total cholesterol (TC) and high-density lipoprotein cholesterol (HDL-C), and glucose level trajectories over the course of a life-time on hypertension development. Therefore, we examined the sex specific trajectories of central adiposity, lipid indices, and glucose level for incident hypertension, using Latent Class Growth Mixture Modeling (LCGMM), in the oldest cohort of the MENA region, namely the Tehran Lipid and Glucose study during a follow-up of more than 12 years.

\section{Methods}

\section{Participants}

Tehran Lipid and Glucose Study (TLGS) is a dynamic prospective population-based study conducted on a representative sample of Tehranians recruited from among residents of district 13 of Tehran. The main groups of ethnicities in Iran are the Caucasians with the most prevalence (75.4\%) and the Turks (22\%). The distribution of ethnicity of Tehran is virtually similar to distribution of ethnicity of Iran [9]. It aims to determine the prevalence and incidence of non-communicable disease risk factors and to develop and implement a healthy lifestyle. The age distribution of the TLGS population at baseline is representative of the overall population of Tehran (Iran National Census, 1996). Details of study design, sampling and rationale hasbeen published elsewhere [10]. In brief, TLGS enrollment was carried out in two phases, the first of which was from January 31, 1999 to July 03, 2001, with a second enrollment phase from October 20, 2001 till September 22, 2005. Data collection is planned to continue for at least 20 years at approximately 3 -year intervals (i.e. phase III: 2005-2008, phase IV: 2008-2011, phase V: 2011-2014, and phase VI: 2015-2018). For the current study, we requited 6750 participants aged $\geq 18$ and $\leq 40$ years (5294 participants from phase 1 and 1456 participants from phase 2). The mean baseline age was 28.87 years (18 to 40 years at baseline and 20 to 60 years at followup). Exclusion criteria included prevalent hypertension $(\mathrm{n}=1056)$ and missing data on BP measurements, WC, TG, HDL-C, TG and FPG ( $\mathrm{n}=300$, considering overlap features between numbers), covariates including BMI, smoking and diabetes status $(\mathrm{n}=56)$, and finally those without any follow-ups after enrolment $(n=308)$ leading to a total of 5030 (2051 of which were male) for data analysis (Additional file 1: Figure S1). Moreover, Based on TLGS protocol waist circumference is not measured for pregnant women. In the current studyWCfor pregnant women were not considered in the analysis if they were pregnant for those relevant phases (i.e., 52, 54, 28, 18 and 8 pregnant women in the follow up 1, 2, 3, 4 , and 5 respectively). WC and TG, HDL-C, TG, FPG data after the onset of hypertension (outcome) were not included in the analyses.

\section{Clinical and laboratory measurements}

Using a standard questionnaire, a trained interviewer collected information including demographic data, drug history and past medical history of diabetes mellitus. Details of anthropometric measurements including weight and height have been reported elsewhere [10]. Weight was measured using digital scale and was recorded to the nearest $100 \mathrm{~g}$, without shoes and with light clothing. Height was measured in a standing position, without shoes. BMIwas calculated by dividing weight $(\mathrm{kg})$ by square of height $(\mathrm{m} 2)$. WC was measured with light clothing at the level of the umbilicus, excluding for pregnant women at each phase. After a 15 -min rest in the sitting position, two measurements of systolic blood pressure (SBP) and diastolic blood pressure (DBP) were measured by trained personnel, on the right arm, using a standardized mercury sphygmomanometer (calibrated by the Iranian Institute of Standards and Industrial Researches); the mean of the two measurements was considered as the participant's BP. A blood sample was taken between 7:00 and 9:00 AM for all study participants, after 12 to $14 \mathrm{~h}$ of overnight fasting. Levels of fasting blood glucose (FBS), total and TG were enzymatically measured [10]. 


\section{Definition of terms}

Hypertension was defined as $\mathrm{SBP} \geq 140 \mathrm{mmHg}$ or $\mathrm{DBP} \geq 90 \mathrm{mmHg}$ or the use ofanti-hypertension medications. BMI was calculated as weight $(\mathrm{kg}) /$ height $(\mathrm{m} 2)$, smoking status was divided into three categories (current/past/never), A current smoker was defined asa person who smokes cigarettes or uses other tobacco products daily or occasionally. A past-smoker was a formerly daily or occasional smoker who currently does not smoke and non-smokers were defined as people who had never smoked. Diabetes mellitus was defined as $\mathrm{FPG} \geq 7 \mathrm{mmol} / \mathrm{L}$ or use of anti-diabetic medications.

\section{Statistical analyses}

Statistical analyses were conducted using the Mplus7.4 (Muthén and Muthén [12]), the SPSS 17.0 and Stata14 software packages. The analyses for the current study consisted of two steps, described in detail below.

\section{Step 1. Latent class growth (mixture) modeling}

Heterogeneity in longitudinal development of WC, TG, HDL-C, TC and FPG was analyzed using Latent Class Growth (Mixture) Modeling (LCGMM) [11, 12]. LCGMM is a contemporaneous longitudinal method based on structural equation modeling, including both continuous and categorical latent variables. The method is an extension of usual growth modeling. In conventional analyses, the theory that all individuals in the study sample come from a single population should hold; meaning that one (average) trajectory will adequately address the developmental pattern of the sample. This hypothesis is relaxed in LCGMM, meaning that individuals in the sample need not come from one single underlying population, but can come from multiple latent sub-clusters.

Finding the number and characteristics of these underlying sub-clusters of individuals is the main goal of LCGMM. This is done by identifying $\mathrm{k}$ number of distinguished latent classes of, in the present study, trajectories of WC, TC, HDL-C, FPG and TG. Each identified class has its own particular growth parameters (intercept, slope), which are also presupposed to be unobserved, or latent. In addition, LCGMM can also account for withinclass variation in either, or both, of the growth parameters, implying within-class heterogeneity on top of the between-class heterogeneity in initial status (intercept) or growth (slope). Varied LCGMM models were run before we chose a final model [13]. First, various linear LCGMM with fixed intercept and slope variance within classes were assessed. Then, quadratic slopes were added to the model permitting for curved developmental patterns. To understand the possibility of different trajectories by gender, these steps were run separately among men and women. Mplus did not compute the standard error in our figures.

\section{Model selection (class identification)}

To identify the optimal number of classes, a common forward procedure was done, starting with a one class solution (i.e., assuming all individuals follow a similar trajectory over time), next adding more classes one at time to examine whether or not the model fit improves due to the additional class. The number of groups were determined by the following criteria: Bayesian information criteria (BIC), entropy, Akaike information criteria (AIC), Adjusted Lo-Mendell-Rubin likelihood ratio test (A LMR test), class size and interpretability [14]. BIC has been shown to be a robust indicator of the preferred $k$ class solution. Lower BIC values indicate better model fit [15]. The AIC evaluates a model or partition scheme by combining the maximum log-likelihood value with a penalty that depends on the number of parameters being estimated. Models that generate the lowest values are optimal [15]. Entropy is an index for assessing the precision of assigning latent class membership. Higher entropy values indicate greater precision of classification [16]. A LMR test assesses the differences in the log-likelihood between LCGA models with $\mathrm{k}$ and $\mathrm{k}+1$ clusters, where p-values $<0.05$ indicates a benefit of adding an additional cluster [17]. We also took the posterior probabilities to assign subjects to the trajectories. Each individual in the sample we reassigned to their most-likely class based on these probabilities which is the highest probability. Therefore, the classes are clearly distinguished from each other. Based on posterior probability, persons were assigned to the trajectory that best matched their work engagement; a probability $>0.8$ is recommended and a probability closer to 1 indicates better classification [14]. For each individual in the sample, the probability of belonging to each of the $\mathrm{k}$ classes is estimated. The probability for the class to which the individual was assigned, should be considerably higher than the other probabilities. By this means, the classes are clearly distinguished from each other. Clinical interpretation and class sample size were also considered in the decision-making process. We rejected models with clinically uninterpretable classes and classes with $<1 \%$ of the total sample. Finally, we considered the usefulness of the separate classes in practice. LCGMM models are computationally-heavy models, often with convergence issues or hitting local maxima. Mplus incorporates random starts to avoid these issues as much as possible. Missing data were adequately handled by the Expectation-Maximization algorithm, which is an iterative method to find maximum 
likelihood estimation with robust standard errors by missing at random (MAR) assumption.

\section{Step 2. Association between the trajectory group membership and incident hypertension}

The first step of the analyses provided us with a classification of the study sample into distinct trajectory subgroups, coded as a categorical variable with $\mathrm{k}$ number of categories. Sex stratify Cox proportional hazard models with follow-up time as the time scale were used to examine the association between trajectory classes of TG, WC, FPG, HDL-C and incident hypertension, considering the trajectory with highest prevalence as reference. We considered multiple potential confounders based on our previous study, and then we included age, SBP, DBP, body mass index (BMI), diabetes, HDL-C, ascovariate. All other statistical tests were two-sided and statistical significance was determined as $\mathrm{p}<0.05$.

\section{Results}

Baseline and end point characteristics are shown in Table 1.

\section{WC, TG, HDL-C, FPG and TC trajectories for Incident BP}

The study population included 2051 males and 2979 females with mean (SD) age of 29.2(6.6) and 33.9(8.4) years, respectively. During follow-up, we found 997 cases (males $=483$ ) with incident hypertension.

\section{WC trajectories}

We performed LCGMM analysis to explore both linear and quadratic terms to fit the model. Model selection between linear and quadratic term was done using CFI, SRMR and TLI; only linear models were applied. Additional file 2: Table $\mathrm{S} 1$ shows the model fit statistics for the growth mixture model with 2 to 6 classes. In both genders, the 4- and 5-class models resulted in some classes that included less than $1 \%$ of the sample, hence, these models were excluded from further consideration. Therefore, the 3-class model was chosen as the optimal class size because it had the lowest BIC, sample size adjusted $\mathrm{BIC}$ and AIC among the remaining models. For females, three waist trajectory classes can be described as: Trajectory 1 (low-increasing, $\mathrm{n}=2606,82.4 \%$ ), Trajectory 2 (High-stable, $\mathrm{n}=275,13.4 \%$ ) and Trajectory 3(highincreasing, $\mathrm{n}=984.2 \%)$.For males, the three waist trajectory classes can be described as: Trajectory 1(stable, $\mathrm{n}=1987$, 94.6\%), Trajectory 2 (low-increasing, $\mathrm{n}=43$, $3.6 \%$ ); and Trajectory 3(high-increasing $=21,1.7 \%$ ) (Fig. 1). Baseline characteristics of the study population by WC trajectory classes are shown in Table 2. Accordingly, among females, participants included in the lowincreasing Trajectory (reference group), generally had
Table 1 characteristics of study population at the baseline and end point*

\begin{tabular}{|c|c|c|c|}
\hline & Baseline & End point & P Value \\
\hline \multicolumn{4}{|c|}{ Females $(n=2979)$} \\
\hline Age (years) & $28.63(6.60)$ & $42.12(7.55)$ & 0.00 \\
\hline \multicolumn{4}{|l|}{ Smoking status } \\
\hline (Current) (\%) & 0.6 & 2.3 & 0.06 \\
\hline (paste) (\%) & 2.3 & 4.1 & \\
\hline BMI (kg/m2) & $25.24(4.66)$ & $28.45(5.10)$ & 0.00 \\
\hline WC (cm) & $80.46(11.32)$ & $91.34(11.62)$ & 0.02 \\
\hline $\mathrm{FPG}(\mathrm{mg} / \mathrm{dL})$ & $87.26(14.43)$ & $94.01(21.06)$ & 0.01 \\
\hline $\mathrm{TG}(\mathrm{mg} / \mathrm{dL})$ & $99(72-143)$ & $111(79-157)$ & 0.04 \\
\hline HDL_C & $44.60(10.30)$ & $50.20(11.47)$ & 0.02 \\
\hline TC & $180(157-206)$ & $186(164-212)$ & 0.05 \\
\hline \multicolumn{4}{|l|}{ Males $(n=2051)$} \\
\hline Age (years) & $29.22(6.67)$ & $42.33(7.58)$ & 0.00 \\
\hline \multicolumn{4}{|l|}{ Smoking status } \\
\hline (Current) (\%) & 26.4 & 29.2 & 0.06 \\
\hline (Paste) (\%) & 9.2 & 13.1 & \\
\hline BMI (kg/m2) & $24.87(4.21)$ & $27.65(4.18)$ & 0.03 \\
\hline WC (cm) & $85.14(11.44)$ & $96.48(10.91)$ & 0.03 \\
\hline $\mathrm{FPG}(\mathrm{mg} / \mathrm{dL})$ & $88(83-94)$ & $96(87-101)$ & 0.04 \\
\hline $\mathrm{TG}(\mathrm{mg} / \mathrm{dL})$ & $133(91-198)$ & $147(103-206)$ & 0.03 \\
\hline $\mathrm{HDL}-\mathrm{C}$ & $37.88(9.05)$ & $41.39(9.84)$ & 0.03 \\
\hline $\mathrm{TC}$ & $182(159-211)$ & $188(164-215)$ & 0.05 \\
\hline
\end{tabular}

Data are mean (standard deviation) for continues, and frequency (\%) for categorical variables *incident hypertension/or the last follow-up

$B M I$ Body mass index, DBP Diastolic blood pressure, SBP Systolic blood pressure, TG Triglyceride, TC total cholesterol, FPG fasting plasma glucose

more favorable baseline characteristics than High-stable and high- increasing rajectories (i.e. lower age, WC, TG, $\mathrm{SBP}$ and DBP). Among males, participants in the stable trajectory (reference group) were older and had the lowest incidence of hypertension. However, in women, the lowest incidence of hypertension was found among participants in the low-increasing trajectory.

As shown in Table 3, among females, compared to the reference group, high stable and high-increasing trajectories were significantly associated with hypertension in the fully adjusted model $[\mathrm{HR}=1.66$ (95\% confidence interval (CI): $((1.26-2.20))],[\mathrm{HR}=2.78$ (95\% confidence interval (CI): (1.79-3.60))]. In women, when we replaced the high-stable with thelow- increasing trajectory as a reference group, the high- increasing trajectory showed a significant risk even in the fully adjusted model (1.49 (1.06-2.11)).

As shown in Table 3, among males, the low-increasing trajectory was significantly associated with hypertension in the fully adjusted model $[\mathrm{HR}=2.76(95 \%$ confidence interval (CI): (1.49-5.10)]. However, the 
a

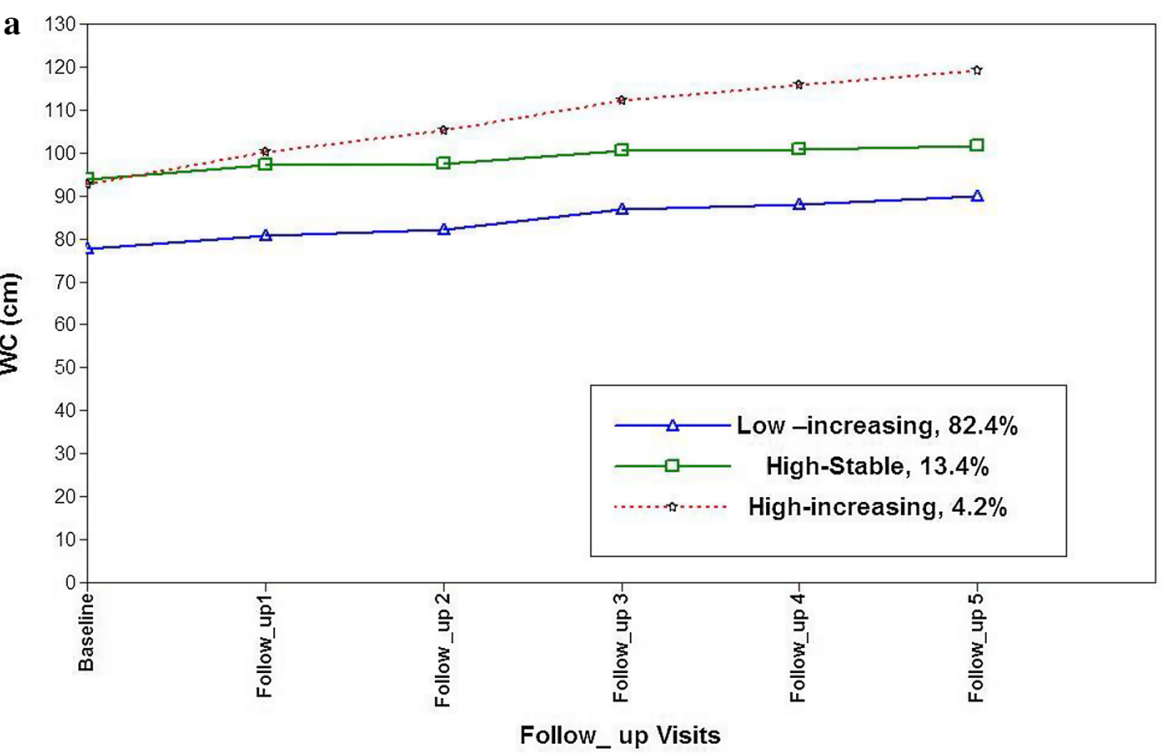

b

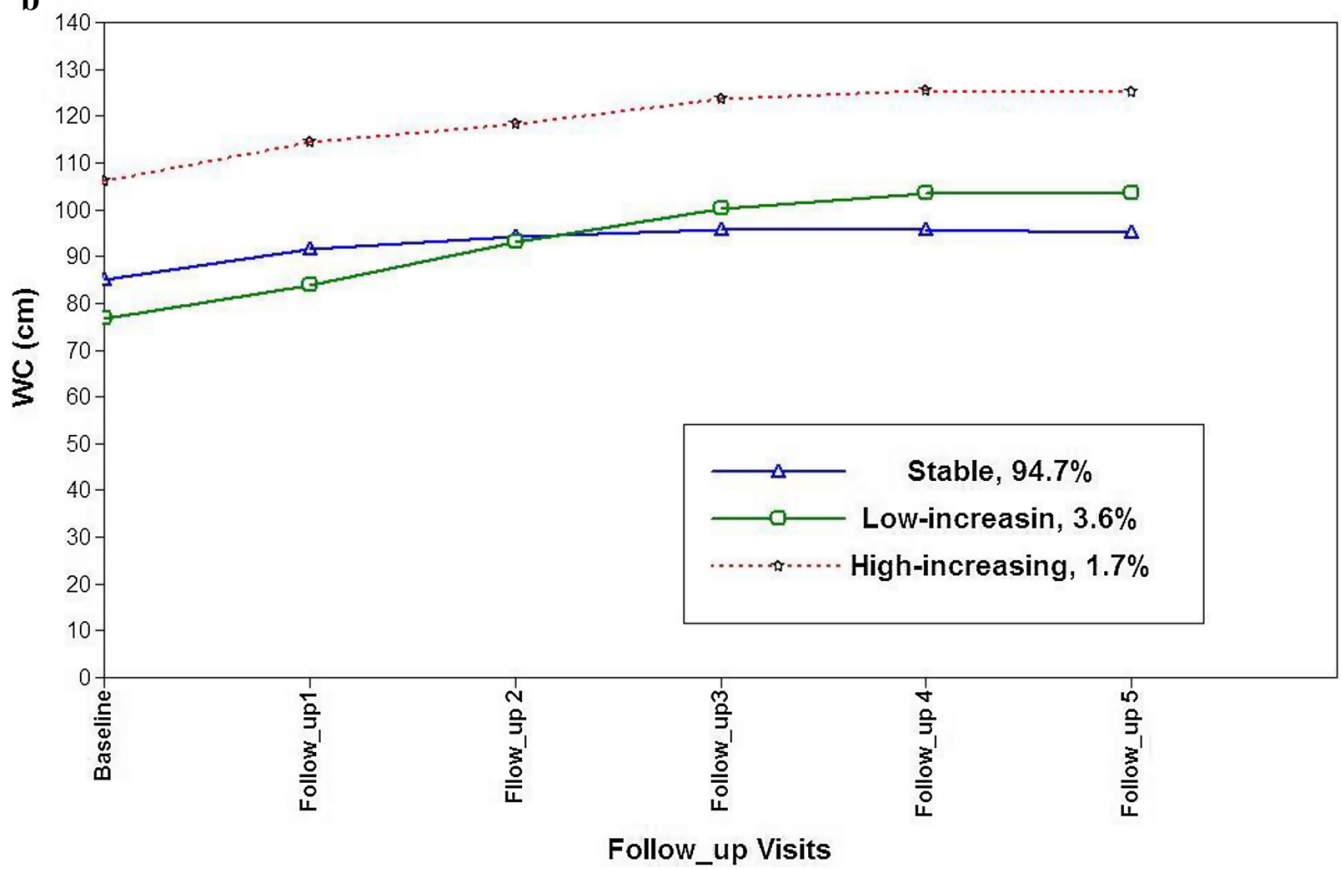

Fig. 1 Linear latent trajectory classes of waist circumference among women for incident hypertension. $\mathbf{b}$ latent trajectory classes of waist circumference among men for incident hypertension

significant association between high-increasing and incident hypertension 2.68 (1.46-4.90) significantly attenuated after adjustment for BMI (Model 2). In men, when we replaced the High-increasing group by the stable group as the reference, the Low-increasing trajectory show higher, but not significant risk in none of the models (Additional file 2: Table S8).

\section{TG Trajectories}

Results of LCGMM analysis showed that the quadratic terms function was the best model. Table 4 shows the quadratic model fit statistics for 2 to 6 classes. As showed in the table, the 4- and 5-class models included less than $1 \%$ of participates and hence were excluded from further consideration. In both genders the 3-class model, was chosen as the optimal class due to the lowest BIC, 
Table 2 Baseline characteristics of study population by waist circumference trajectories for incident hypertension

\begin{tabular}{|c|c|c|c|c|}
\hline Females & Low-increasing $(n=2606)$ & High-Stable $(n=275)$ & High-increasing $(n=98)$ & $P$ value \\
\hline Age (years) & $28.15(6.60)$ & $32.78(5.17)$ & $29.52(5.93)$ & 0.000 \\
\hline Smoking status & & & & 0.04 \\
\hline Current) (\%) & 0.7 & 0.4 & 0.0 & \\
\hline (Paste) (\%) & 2.1 & 4.7 & 4.1 & \\
\hline $\mathrm{BMI}(\mathrm{kg} / \mathrm{m} 2)$ & $24.21(3.77)$ & $32.56(3.4)$ & $32.01(5.25)$ & 0.000 \\
\hline WC (cm) & $77.93(9.17)$ & $95.00(7.13)$ & $95.04(8.01)$ & 0.000 \\
\hline Diabetes, n (\%) & $20(0.8)$ & $12(4.4)$ & 0 & 0.000 \\
\hline $\mathrm{TG}(\mathrm{mg} / \mathrm{dL})$ & $95(70-133)$ & $148(109-206)$ & $125(83-174)$ & 0.000 \\
\hline $\mathrm{SBP}(\mathrm{mmHg})$ & $106.7(10.26)$ & $111.28(9.70)$ & $111.08(9.53)$ & 0.000 \\
\hline $\mathrm{DBP}(\mathrm{mmHg})$ & $71.91(8.12)$ & $76.1(7.18)$ & $75.77(6.94)$ & 0.000 \\
\hline Hypertension, n (\%) & $362(13.9)$ & $104(37.8)$ & $48(49)$ & 0.000 \\
\hline Males & Low- increasing $(n=43)$ & Stable $(n=1987)$ & High-increasing $(n=21)$ & $P$ value \\
\hline Age (years) & $22.16(5.39)$ & $29.40(6.61)$ & $26.95(6.32)$ & 0.000 \\
\hline Smoking status & & & & 0.2 \\
\hline (Current) (\%) & 18.6 & 26.4 & 14.3 & \\
\hline (Paste) (\%) & 4.7 & 9.8 & 4.8 & \\
\hline BMI (kg/m2) & $20.82(2.42)$ & $24.85(4.05)$ & $35.4(4.67)$ & 0.000 \\
\hline WC (cm) & $73.72(6.30)$ & $85.11(11.07)$ & $111.42(12.56)$ & 0.000 \\
\hline Diabetes, n (\%) & 0 & $28(1.4)$ & 0 & 0.04 \\
\hline $\mathrm{TG}(\mathrm{mg} / \mathrm{dL})$ & $89(70-113)$ & $135(91-200)$ & $164(122-217)$ & 0.000 \\
\hline $\mathrm{SBP}(\mathrm{mmHg})$ & $105.48(11.72)$ & $112.39(10.42)$ & $121.76(9.74)$ & 0.000 \\
\hline $\mathrm{DBP}(\mathrm{mmHg})$ & $70.46(9.50)$ & $73.77(8.13)$ & 77.47 (9.88) & 0.000 \\
\hline Hypertension, n (\%) & $12(27.9)$ & $460(23.2)$ & $11(52.4)$ & 0.006 \\
\hline
\end{tabular}

Data are mean (standard deviation) for continuous, and frequency (\%) for categorical variables. BMI Body mass index, DBP Diastolic blood pressure, SBP Systolic blood pressure, TG Triglyceride, WC Waist circumference

Table 3 Associations between waist circumference trajectories and hypertension

\begin{tabular}{|c|c|c|c|c|c|c|c|c|c|}
\hline & \multicolumn{3}{|l|}{ Model1 } & \multicolumn{3}{|l|}{ Model2 } & \multicolumn{3}{|l|}{ Model3 } \\
\hline & Adjusted HR (95\% Cl) & $\beta$ & $P$ value & Adjusted HR (95\% Cl) & $\beta$ & $P$ value & Adjusted HR (95\% Cl) & $\beta$ & $P$ value \\
\hline \multicolumn{10}{|l|}{ Females } \\
\hline Low-increasing & Reference & & & Reference & & & Reference & & \\
\hline High-stable & $2.21(1.76-2.76)$ & .79 & 0.000 & $1.68(1.27-2.21)$ & .51 & 0.000 & $1.67(1.26-2.20)$ & 0.50 & 0.000 \\
\hline High-increasing & $3.28(2.43-4.45)$ & 1.19 & 0.000 & $2.50(1.76-3.50)$ & .91 & 0.000 & $2.78(1.79-3.60)$ & 0.93 & 0.000 \\
\hline \multicolumn{10}{|l|}{ Males } \\
\hline Stable & Reference & & & Reference & & & Reference & & \\
\hline Low-increasing & $2.47(1.38-4.43)$ & 0.90 & 0.002 & $2.85(1.58-5.13)$ & 1.04 & 0.002 & $2.76(1.49-5.10)$ & 1.01 & 0.001 \\
\hline High-increasing & $2.68(1.46-4.90)$ & 0.98 & 0.001 & $1.48(0.76-2.89)$ & 0.39 & 0.24 & $1.44(0.74-2.81)$ & 0.36 & 0.28 \\
\hline
\end{tabular}

Cl Confidence interval, $H R$ Hazard ratio

Model1 was adjusted for age, SBP, DBP

Model 2 was adjusted for age, SBP, DBP, BMI

Model3: was adjusted for age, SBP, DBP, Smoking Statu, Diabetes, BMI, HDL-C

sample size adjusted BIC and AIC between the remaining models (Additional file 2: Table S3). In females, Trajectory 1 (stable, $\mathrm{n}=2740,91.3 \%$ ), Trajectory 2 (decreasing, $\mathrm{n}=158,5.9 \%$ ) and Trajectory 3 (inverse $\mathrm{U}$-shape, $\mathrm{n}=81$, $2.8 \%$ ) were found. In males Trajectory 1 (stable, $\mathrm{n}=2740$,
89.7\%), Trajectory 2(inverse U-shape, $\mathrm{n}=121,6.2 \%$ ), Trajectory 3(decreasing, $\mathrm{n}=85,4.2 \%$ ) were identified (Fig. 2). Baseline characteristics of the study population by waist trajectory classes are shown in Table 4. Accordingly, among females, participants included in the stable 
Table 4 Baseline characteristics of study population by triglyceride trajectories for incident hypertension

\begin{tabular}{|c|c|c|c|c|}
\hline Females & Stable $(n=2740)$ & Inverse U-shape $(n=81)$ & Decreasing $(n=158)$ & $P$ value \\
\hline Age (years) & $28.26(6.57)$ & $29.52(5.93)$ & $32.78(5.77)$ & 0.000 \\
\hline \multicolumn{5}{|l|}{ Smoking status } \\
\hline (Current) (\%) & 0.5 & 2.9 & 0.6 & \\
\hline (Paste) (\%) & 2.2 & 3.7 & 3.8 & \\
\hline $\mathrm{BMI}(\mathrm{kg} / \mathrm{m} 2)$ & $24.98(4.59)$ & $26.99(4.32)$ & $28.80(4.22)$ & \\
\hline$W C(\mathrm{~cm})$ & 79.73 (11.06) & 86.85 (10.73) & $90.52(10.13)$ & 0.000 \\
\hline Diabetes n (\%) & $18(0.7)$ & $6(7.4)$ & $8(5.1)$ & 0.000 \\
\hline $\mathrm{TG}(\mathrm{mg} / \mathrm{dL})$ & $95(70-133)$ & $128(168-233)$ & $290(260-324)$ & 0.000 \\
\hline $\mathrm{SBP}(\mathrm{mmHg})$ & $106.96(10.17)$ & $108.08(10.53)$ & $113.25(10.30)$ & 0.000 \\
\hline $\mathrm{DBP}(\mathrm{mmHg})$ & 72.07 (8.09) & $74.67(7.54)$ & 77.16 (6.88) & 0.000 \\
\hline $\mathrm{BP}, \mathrm{n}(\%)$ & $424(15.5)$ & $32(39)$ & $58(36.7)$ & 0.000 \\
\hline Males & Stable $(n=1845)$ & Inverse U-shape $(n=121)$ & Decreasing $(n=85)$ & $P$ value \\
\hline Age (years) & $29.04(6.76)$ & $28.70(6.34)$ & $33.89(4.39)$ & 0.000 \\
\hline \multicolumn{5}{|l|}{ Smoking status } \\
\hline (Current) (\%) & 25.4 & 32.2 & 32.9 & \\
\hline (Paste) (\%) & 9.3 & 8.3 & 9.4 & \\
\hline BMI (kg/m2) & $24.68(4.17)$ & $25.3(4.13)$ & $28.27(3.51)$ & 0.01 \\
\hline$W C(\mathrm{~cm})$ & $84.61(11.36)$ & 85.89 (11.33) & $94.75(9.11)$ & 0.000 \\
\hline Diabetes, n (\%) & $28(1.1)$ & $2(1.7)$ & $6(7.1)$ & 0.04 \\
\hline $\mathrm{TG}(\mathrm{mg} / \mathrm{dL})$ & 124 (87-181) & $215(144-288)$ & $467(409-571)$ & 0.000 \\
\hline $\mathrm{SBP}(\mathrm{mmHg})$ & $112.06(10.48)$ & $113.43(11.6)$ & 116.92 (9.52) & 0.000 \\
\hline $\mathrm{DBP}(\mathrm{mmHg})$ & $73.52(8.14)$ & 73.55 (8.1) & 78.67 (8.12) & 0.000 \\
\hline $\mathrm{BP}, \mathrm{n}(\%)$ & $416(22.5)$ & $32(26.4)$ & $35(41.2)$ & 0.000 \\
\hline
\end{tabular}

Data are mean (standard deviation) for continues, and frequency (\%) for categorical variables

$B M I$ Body mass index, DBP Diastolic blood pressure, SBP Systolic blood pressure; TG Triglyceride

trajectory generally had more favorable baseline characteristics than inverse U-shape and decreasing trajectories (i.e. lower age, WC, FPG, TG, SBP and DBP). Among men (similar to women) participants in the stable trajectory had more favorable baseline characteristics than other trajectories, excluding participants' age which was lower in the inverse U-shape trajectory. In both genders, the lowest incidence of hypertension was found among participants in the stable trajectory.

As shown in Table 5, among females, compared to the stable trajectory as a reference group, the inverse U-shape and decreasing trajectories were significantly associated with hypertension in the fully adjusted model $[\mathrm{HR}=1.36$ (95\% confidence interval $(\mathrm{CI}):((1.02-1.80))]$, $[\mathrm{HR}=2.25$ (95\% confidence interval (CI): (1.58-3.27)),]. When we replaced the decreasing trajectory with the stable trajectory as a reference group, the inverse U-shape trajectory showed a significant risk even in the fully adjusted model (1.64 (1.05-2.54)). As shown in Table 5, among males, a $43 \%$ increased risk of hypertension in the decreasing trajectory (1.01-2.04) significantly attenuated after adjusting for BMI (Model 2). In men when we replaced the High- increasing group by the stable group as the reference, the Low -increasing trajectory did not show higher significant risk in none of the models.

\section{HDL-C, FPG and TC trajectories}

We performed LCGMM analysis to explore both linear and quadratic terms to fit the model. Model selection between linear and quadratic term was done using CFI, SRMR and TLI; only linear models were applied. For HDL-C, FPG. In both genders, the 2-class model was chosen as the optimal class size because it had the lowest BIC, sample size adjusted BIC and AIC among the remaining models. For TC in both gender all individuals followed a similar trajectory and 1-class model was chosen as the optimal class. For females, tow FPG trajectory classes can be described as: Trajectory 1 (Stable, $n=2927$, 98.3\%), Trajectory 2 (increasing, $\mathrm{n}=52,1.7 \%$ ) and tow HDL-C trajectory classes can be described as: Trajectory 1 (Low increasing, $\mathrm{n}=2759,89.6 \%$ ), Trajectory 2 (High increasing, $\mathrm{n}=220,10.4 \%)$ one TC trajectory can be described as Trajectory 1 (Stable, $\mathrm{n}=2979,100 \%$ ).

For males, the tow FPG trajectory classes can be described as: Trajectory 1 (stable, $\mathrm{n}=1980,96.5 \%$ ), Trajectory 2 (increasing, $\mathrm{n}=71,3.6 \%$ ), tow HDL-C 


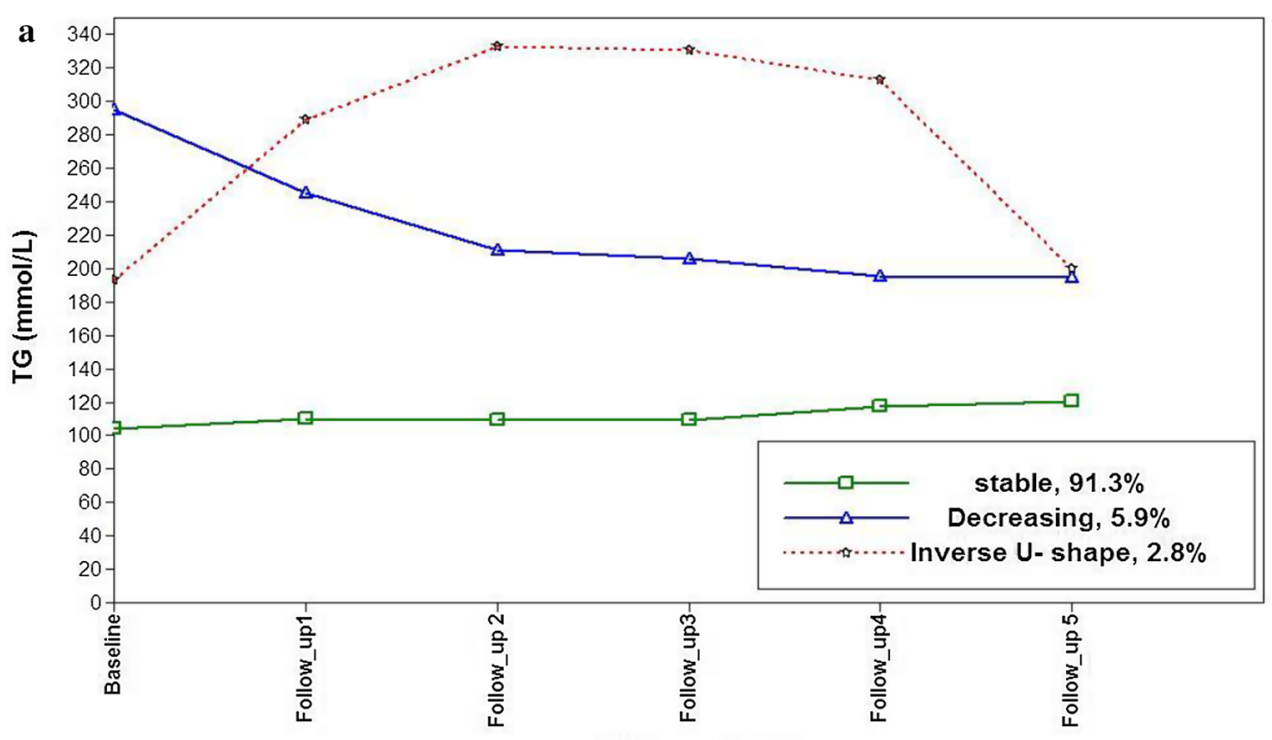

Follow_up Visits

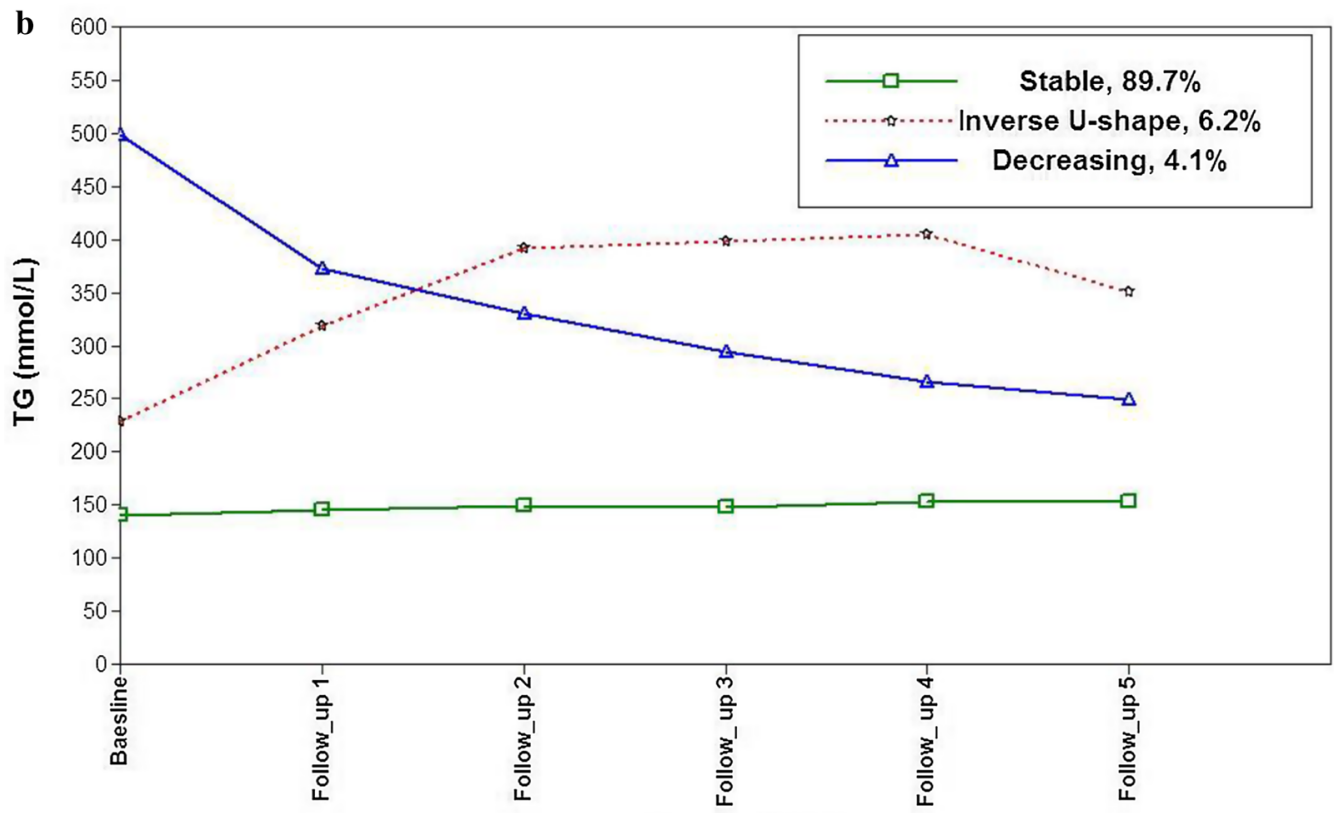

Follow-up Visits

Fig. 2 a Quadratic latent trajectory classes of triglycerides among women for incident hypertension. b Quadratic latent trajectory classes of triglycerides among men for incident hypertension

trajectory classes can be described as: Trajectory 1(Low increasing, $n=1913,91.6 \%$ ), Trajectory 2 (High increasing, $\mathrm{n}=131,8.4 \%$ ) one TC trajectory can be described as Trajectory 1 (Stable, $\mathrm{n}=2051,100 \%$ ) Additional file 3, Additional file 4, Additional file 5. Baseline characteristics of the study population by FPG, HDL-C and TC trajectory classes are shown in Additional file 2: Tables S3, S5 and S7. As shown in Additional file 2: Tables S4, S6, we did not find any impact of increasing level of FPG and HDL-C on incident hypertension compared with their reference groups. Considering TC, all individuals followed a similar trajectory. 
Table 5 Associations between Triglyceride trajectories and Hypertension

\begin{tabular}{|c|c|c|c|c|c|c|c|c|c|}
\hline & \multicolumn{3}{|l|}{ Model1 } & \multicolumn{3}{|l|}{ Model2 } & \multicolumn{3}{|l|}{ Model3 } \\
\hline & Adjusted HR (95\% Cl) & $\beta$ & $P$ value & Adjusted HR (95\% Cl) & $\beta$ & $P$ value & Adjusted HR (95\% Cl) & $\beta$ & P value \\
\hline \multicolumn{10}{|l|}{ Females } \\
\hline Stable & Reference & & & Reference & & & Reference & & \\
\hline Inverse U-shape & $2.45(1.70-3.51)$ & 0.89 & 0.000 & $2.27(1.57-3.26)$ & 0.82 & 0.000 & $2.23(1.58-3.23)$ & 0.80 & 0.000 \\
\hline Decreasing & $1.45(1.09-1.93)$ & 0.37 & 0.009 & $1.36(1.02-1.80)$ & 0.03 & 0.031 & $1.32(1.01-1.76)$ & 0.30 & 0.033 \\
\hline \multicolumn{10}{|l|}{ Males } \\
\hline Stable & Reference & & & Reference & & & Reference & & \\
\hline Inverse U shape & $1.07(0.74-1.52)$ & 0.07 & 0.700 & $1.05(0.73-1.50)$ & 0.28 & 0.77 & $1.09(0.75-1.55)$ & 0.09 & 0.69 \\
\hline Decreasing & $1.43(1.01-2.04)$ & 0.36 & 0.042 & $1.33(0.93-1.88)$ & 0.52 & 0.11 & $1.36(0.95-1.89)$ & 0.30 & 0.08 \\
\hline
\end{tabular}

Cl Confidence interval, $H R$ Hazard ratio

Model 1 was adjusted for age, SBP, DBP

Model 2was adjusted for age, SBP, DBP, BMI

Model 3: was adjusted for age, SBP, DBP, Smoking Status, Diabetes, BMI, HDL-C

\section{Discussion}

To our knowledge, this is the first population-based study that examined the sex-specific trajectories of central adiposity (as assessed by WC), dyslipidemia (as assessed by TG, HDL-C, and TC level), and FPG for incident hypertension among the middle-aged adult population, during more than a decade offollow-up. Using the LCGM method, 3 distinct groups of WC and TG, 2 distinct groups of HDL-C and FPG, and only one group of $\mathrm{TC}$ changes were found in each gender. In addition, we examined the associations of these trajectory groups with incident hypertension in different models.

\section{WC trajectory and incident hypertension}

For WC in our study, among women in the high-stable group (consisting of about 13\% of the total population) waist circumference stayed stable at around $95 \mathrm{~cm}$, while the WC of the other two groups (about $87 \%$ of total population) increased with different baseline levels and incremental slopes. Interestingly, among women, the majority of our cohort (low-increasing trajectory, reference group) showed the increasing value of $\mathrm{WC}$, but incident hypertension was low in that group; the issue might be attributable to that this value did not capture the threshold of $90 \mathrm{~cm}$, the cut-off point for the development of hypertension in both genders among Iranian population [18]. The increasing trend of high WC might be attributed to progressive decrease in physical activity level among Iranian women mainly related to decrease in work-related activity [19]. We found that female participants in the highincreasing group showed a more than 2.5 -fold increased risk of hypertension compared to the reference group, after adjustment for age, BMI, SBP, DBP, smoking status, diabetes and HDL-C. Although participants in the high-stable and high-increasing groups had the same intercept for $\mathrm{WC}$, but when considering the high stable group as reference, participants in the high-increasing group showed a $50 \%$ higher risk of hypertension.

Among men, WC trajectories had a steeper initial WC increase in about $5 \%$ of the population, however, the majority had stable WC levels over time. Participants in the low-increasing group (i.e. WC 78 at the onset and $103 \mathrm{~cm}$ at last follow-up) showed an approximately 2.5fold increased risk of hypertension compared to those in the stable group. However, for the high- increasing group with a mean BMI of around $35 \mathrm{~kg} / \mathrm{m}^{2}$ and WC of 107 at onset, this risk was not independent of baseline BMI; this indicates that increasing WC does not have significant role in predicting hypertension in individuals with moderate level of obesity at baseline. Actually, general adiposity remained as an independent risk factor in the model [hazard ratio of BMI $=1.10$ (CI 1.2-1.48)]. No literature exists concerning the risk of hypertension in men with a baseline BMI greater than $32 \mathrm{~kg} / \mathrm{m}^{2}$.

We demonstrated that increasing WC values are generally associated with hypertension incidence after adjustment for confounders (especially BMI), irrespective of the baseline values of WC which is in line with the results of previous longitudinal population-based studies [2022]. The Iranian National Committee of Obesity defined WC cut-offs of $90 \mathrm{~cm}$ in both genders as the cut-off point for screening for CVD risk factors including hypertension and diabetes [18]. In the current study, both the trajectories that showed higher risk of hypertension among women had a WC of $95 \mathrm{~cm}$ at baseline, however, the high increasing trajectory that had the greatest risk of hypertension showed a higher slope in WC. Similarly, among men, in the low-increasing group with normal BMI at baseline and higher slope in $\mathrm{WC}$, showed significant risk 
for incident hypertension after controlling for BMI. In a representative population-based study among the Italian population, using two point measurements over about a decade, in a model adjusting for confounders, the risk of office, home, and 24-h hypertension for an increase of $1 \mathrm{~cm}$ of WC were $1.8 \%, 1.5 \%$ and $1.4 \%$, respectively (all $\mathrm{p}$ values $<0.05$ ) [23]. Moreover, among both Chinese men and women, those with or without abdominal obesity at baseline who had abdominal obesity at follow-up of 6 years showed significant risk for hypertension development independent of a large set of covariates (including baseline level of BMI) [22].

Increasing levels of $\mathrm{WC}$ apart from increasing insulin resistance can lead to hypertension through several well-known mechanisms [24]. Excessive fat tissues can contribute to sympathetic nervous system activation and secretion of molecules with the capacity of promoting the generation of aldosterone and angiotensin II, whose vasopressor and anti-natriuretic activities have been well-documented [24].

\section{TG trajectory and incident hypertension}

We found similar trajectory patterns of TG trends for hypertension risk among both men and women. Approximately $90 \%$ of individuals of both genders stayed in the stable group over time. In the present study, only women with inverse U-shape or decreasing trajectories showed significant risk for incident hypertension in the fully adjusted model compared to the stable trajectory. Moreover, inverse U-shaped trajectories, comprising only about 3\% of our population and having lower TG values at baseline compared to the decreasing trajectory (188 vs $298 \mathrm{mg} / \mathrm{dl}$ ), showed significantly higher risk for incident hypertension in all three adjusted models. This phenomenon underlines not only the importance of the TG slope, but the superiority of the TG slope in predicting hypertension incidence compared to TG at intercept as well. Albeit several studies have affirmed that TG at baseline is a crucial factor for hypertension development $[25,26]$, no study, until now, has evaluated the association between hypertension incidence and TG trajectory.

In our study the association between TG trajectories and hypertension incidence among men disappeared after controlling for BMI, hence, it can be concluded that TG in men does not play an independent role in inducing hypertension; an issue that was previously found among TLGS participants using snapshot measurements of TG at baseline recruitment $[27,28]$. The result of Zhang et al's study is similar to our results, highlighting the importance of TG in hypertension incidence especially among women [29]. However, an association between TG levels and incident hypertension in both sex groups independent of adiposity were shown in the Spanish population [30]. We previously demonstrated that during the last decade, obesity is showing an increasing trend, however, there is a decreasing trend for TG and TC concentrations among the Iranian population [35, 36]. This paradoxical situation is not restricted to the Iranian population and it was repeatedly shown in American as well as European populations, frequency of obesity is at an increasing pace whereas TG trend was downward in men and women [37-39]. Even in our data analysis among those with Inverse U-shape TG trajectory, when we examined changes of WC in this group, WC increased from 85 to 93 and 85 to 97 in women and men, respectively, opposite to the TG changes (Additional file 6: Figure S5).

Although explaining the mechanism behind rising BP in individuals with increasing levels of TG was not the goal of the present study, a number of studies have attempted to clarify this issue, nevertheless, the exact pathophysiology mechanism has yet to be determined. An unfavorable lipid profile disturbs endothelial function, which leads to a variety of complications including impairment in vasoregulation [31], decrease in the compliance of arteries, increase in arterial stiffness [32], and renal dysfunction [33].In addition to these, ample evidence in confirmation of the role of dyslipidemia (especially high levels of TG) in insulin resistance which maintains a mandatory role in the pathogenesis of hypertension, provide the most plausible explanation for the mechanism of hypertension incidence in individuals with high levels of TG [34]. Currently, as far as we know, no study has assessed the reason behind inter-sex differences with regards to the potential role of TG in inducing hypertension. However, Zhang et al. speculated that this may be because of differences in sex hormones [29].

\section{HDL-C, FPG, and TC trajectories and incident hypertension}

In the present study, For HDL-C, the trajectory patterns in men and women were alike. Both trajectories (Lowincreasing and High-increasing) had increasing pattern with different intercepts. Regarding FPG, similar to HDL$\mathrm{C}$, men and women had the same trajectory patterns. In both gender, the majority of our cohort (Stable trajectory, reference group) stayed stable at around $90 \mathrm{mg} / \mathrm{dL}$. It is noteworthy that only one pattern (Stable trajectory) was detected for men and women of the present study. As far as we are aware, no study has evaluated the correlations between HDL-C, TC, and FPG trajectories and incident hypertension. We did not find any significant associations between increasing values of HDL-C and FPG and incident hypertension.

We acknowledge that our study had some strengths. First, to the best of our knowledge, this is the only study 
that assessed the association between sex-specific WC and TG trajectories and hypertension incidence. Second, the association between TG and hypertension incident cannot be fully reliable if the length of the follow-up is short [26], hence, owing to the long duration of follow-up in the current study, our results can be trusted. Finally, although the association between hypertension and BMI in individuals with BMI above $32 \mathrm{~kg} / \mathrm{m}^{2}$ has not yet been examined [40], we found that in individuals with a mean BMI of $35.4 \mathrm{~kg} / \mathrm{m}^{2}$, BMI has a strong association with hypertension incidence and has superiority over WC in predicting the risk of hypertension.

As a limitation, while we adjusted three models for a variety of covariates, there is still a possibility of residual confounding. Nutritional status and physical activity play an important role in evaluating hypertension risk. Unfortunately, we did not have data regarding nutritional status for TLGS participants at the baseline recruitment. Moreover, since the tools for assessing physical activity level changed between the two baseline recruitments, we did not consider this covariate in our data analysis [10]. Third, this study was conducted in the metropolitan city of Tehran, hence, our findings might not be generalizable to rural areas.

During more than a decade of follow-up, we found significant differences between genders in the association between trajectories of WC and TG with incident hypertension using LCGMM. Accordingly, after controlling for traditional risk factors and BMI among women, both a higher value of $\mathrm{WC}$ at the baseline and particularly a higher slope, were strongly associated with greater risk of hypertension. However, among men, this higher risk for increasing trajectories of WC was limited to men with normal BMI at baseline. Furthermore, only among women, a trajectory with fast increasing TG value, despite lower levels at baseline, was strongly associated with hypertension incidence.

\section{Supplementary Information}

The online version contains supplementary material available at https://doi. org/10.1186/s12967-021-02749-x.

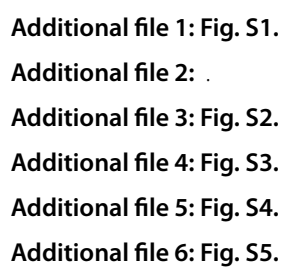

\section{Abbreviations}

WC: Waist circumference; TG: Triglyceride; BP: Blood pressure; SBP: Systolic blood pressure; DBP: Diastolic blood pressure; BMI: Body mass index; FPG: Fasting plasma glucose; FBS: Fasting blood glucose; TLGS: Tehran Lipid and Glucose Study; LCGMM: Latent Class Growth Mixture Modeling.

\section{Acknowledgements}

We would also like to express our appreciation to the research team members and to TLGS participants for their enthusiastic support.

\section{Authors' contributions}

$\mathrm{NA}$ and $\mathrm{FH}$ : study conception and design; FA, FH, and DK: acquisition of the data; NA: Analysis and interpretation of data; NA and SSTZ; writing the first draft. FH and DK; critical revision. All authors have read and approved the final manuscript.

\section{Funding}

There is no funding disclosure to declare.

\section{Availability of data and materials}

The datasets used and/or analyzed during the current study are available from the corresponding author on reasonable request.

\section{Ethics approval and consent to participate}

All participants gave written informed consents according to the Helsinki Declaration guideline and the study was approved by the ethics committee of the Research Institute for Endocrine Science.

\section{Consent for publication}

All authors reviewed and approved the final version for submission.

\section{Competing interests}

There are no competing interests concerning the publication of the current article.

\section{Author details}

1 Prevention of Metabolic Disorders Research Center, Research Institute for Endocrine Sciences, Shahid Beheshti University of Medical Sciences, No. 24, Parvaneh Street, Velenjak, Tehran, Iran. ${ }^{2}$ School of Medicine, Tehran University of Medical Sciences, Tehran, Iran. ${ }^{3}$ Department of Biostatistics and Epidemiology, Research Institute for Endocrine Sciences, Shahid Beheshti University of Medical Sciences, Tehran, Iran. ${ }^{4}$ Endocrine Research Center, Research Institute for Endocrine Sciences, Shahid Beheshti University of Medical Sciences, Tehran, Iran.

Received: 31 October 2020 Accepted: 11 February 2021

Published online: 23 February 2021

\section{References}

1. Fahed AC, El-Hage-Sleiman A-KM, Farhat TI, Nemer GM. Diet, genetics, and disease: a focus on the Middle East and North Africa region. Journal of Nutrition and Metabolism. 2012.

2. Kearney PM, Whelton M, Reynolds K, Muntner P, Whelton PK, He J. Global burden of hypertension: analysis of worldwide data. Lancet. 2005;365(9455):217-23.

3. Kalantari S, Khalili D, Asgari S, Fahimfar N, Hadaegh F, Tohidi M, et al. Predictors of early adulthood hypertension during adolescence: a population-based cohort study. BMC Public Health. 2017;17(1):915.

4. Asgari S, Khalili D, Mehrabi Y, Kazempour-Ardebili S, Azizi F, Hadaegh F. Incidence and risk factors of isolated systolic and diastolic hypertension: a 10 year follow-up of the Tehran Lipids and Glucose Study. Blood Press. 2016;25(3):177-83.

5. Echouffo-Tcheugui JB, Batty GD, Kivimäki M, Kengne AP. Risk models to predict hypertension: a systematic review. PLoS ONE. 2013;8(7):e67370.

6. Sun D, Liu J, Xiao L, Liu Y, Wang Z, Li C, et al. Recent development of risk-prediction models for incident hypertension: An updated systematic review. PLoS ONE. 2017;12(10):e0187240.

7. Ahanchi NS, Ramezankhani A, Munthali RJ, Asgari S, Azizi F, Hadaegh F. Body mass index trajectories from adolescent to young adult for incident high blood pressure and high plasma glucose. PLoS ONE. 2019;14(5):e0213828.

8. Fan B, Yang Y, Dayimu A, Zhou G, Liu Y, Li S, et al. Body mass index trajectories during young adulthood and incident hypertension: a longitudinal cohort in Chinese population. J American Heart Association. 2019;8(8):e011937. 
9. Moghimi N, Davatchi F, Rahimi E, Saidi A, Rashadmanesh N, Moghimi S, et al. WHO-ILAR COPCORD study (stage 1, urban study) in Sanandaj. Iran Clinical Rheumatol. 2015;34(3):535-43.

10. Azizi F, Ghanbarian A, Momenan AA, Hadaegh F, Mirmiran P, Hedayati $M$, et al. Prevention of non-communicable disease in a population in nutrition transition: Tehran Lipid and Glucose Study phase II. Trials. 2009;10(1):5.

11. Muthén B. The potential of growth mixture modelling. Infant and Child Development. 2006;15(6):623.

12. Muthén L, Muthén B. Mplus statistical modeling software (Version 6.12). Los Angeles: Muthén \& Muthén. 2011.

13. Berlin KS, Parra GR, Williams NA. An introduction to latent variable mixture modeling (part 2): longitudinal latent class growth analysis and growth mixture models. J Pediatr Psychol. 2014;39(2):188-203.

14. Nylund KL, Asparouhov T, Muthén BO. Deciding on the number of classes in latent class analysis and growth mixture modeling: A Monte Carlo simulation study. Structural Equation Modeling A multidisciplinary J. 2007;14(4):535-69.

15. Wang M, Bodner TE. Growth mixture modeling: Identifying and predicting unobserved subpopulations with longitudinal data. Organizational Research Methods. 2007;10(4):635-56.

16. Jung T, Wickrama KA. An introduction to latent class growth analysis and growth mixture modeling. Soc Pers Psychol Compass. 2008;2(1):302-17.

17. McLachlan GJ, Peel D. Finite mixture models: John Wiley \& Sons; 2004.

18. AZIZI F, Khalili D, Aghajani H, ESTEGHAMATI A, Hosseinpanah F, DELAVARI $A$, et al. Appropriate waist circumference cut-off points among Iranian adults: the first report of the Iranian National Committee of Obesity. 2010.

19. Koohpayehzadeh J, Etemad K, Abbasi M, Meysamie A, Sheikhbahaei S, Asgari $F$, et al. Gender-specific changes in physical activity pattern in Iran: national surveillance of risk factors of non-communicable diseases (2007-2011). International J Public Health. 2014:59(2):231-41.

20. Chuang S-Y, Chou P, Hsu P-F, Cheng H-M, Tsai S-T, Lin I-F, et al. Presence and progression of abdominal obesity are predictors of future high blood pressure and hypertension. Am J Hypertens. 2006;19(8):788-95.

21. Miyatake N, Matsumoto S, Fujii M, Numata T. Reducing waist circumference by at least $3 \mathrm{~cm}$ is recommended for improving metabolic syndrome in obese Japanese men. Diabetes Res Clin Pract. 2008;79(2):191-5.

22. Zhao Y, Zhang M, Luo X, Wang C, Li L, Zhang L, et al. Association of 6-year waist circumference gain and incident hypertension. Heart. 2017;103(17):1347-52

23. Bombelli M, Facchetti R, Sega R, Carugo S, Fodri D, Brambilla G, et al. Impact of body mass index and waist circumference on the long-term risk of diabetes mellitus, hypertension, and cardiac organ damage. Hypertension. 2011;58(6):1029-35.

24. Poirier P, Lemieux I, Mauriege P, Dewailly E, Blanchet C, Bergeron J, et al. Impact of waist circumference on the relationship between blood pressure and insulin: the Quebec Health Survey. Hypertension. 2005;45(3):363-7.

25. Hunt SC, Stephenson SH, Hopkins PN, Williams RR. Predictors of an increased risk of future hypertension in Utah. A screening analysis. Hypertension. 1991;17(6_pt_2):969-76.

26. Otsuka T, Takada H, Nishiyama Y, Kodani E, Saiki Y, Kato K, et al. Dyslipidemia and the risk of developing hypertension in a working-age male population. J American Heart Association. 2016;5(3):e003053.
27. Asgari S, Moazzeni SS, Azizi F, Abdi H, Khalili D, Hakemi MS, et al. SexSpecific Incidence Rates and Risk Factors for Hypertension During 13 Years of Follow-up: The Tehran Lipid and Glucose Study. Global Heart. 2020;15(1).

28. Tohidi M, Hatami M, Hadaegh F, Azizi F. Triglycerides and triglycerides to high-density lipoprotein cholesterol ratio are strong predictors of incident hypertension in Middle Eastern women. J Hum Hypertens. 2012;26(9):525

29. Zhang L, Li J-I, Zhang L-I, Guo L-I, Li H, Li D. Association and interaction analysis of body mass index and triglycerides level with blood pressure in elderly individuals in China. BioMed research international. 2018;2018.

30. Sánchez-l̂̃igo L, Navarro-González D, Pastrana-Delgado J, FernándezMontero A, Martínez JA. Association of triglycerides and new lipid markers with the incidence of hypertension in a Spanish cohort. J Hypertens. 2016;34(7):1257-65.

31. Selwyn AP, Kinlay S, Libby P, Ganz P. Atherogenic lipids, vascular dysfunction, and clinical signs of ischemic heart disease. Circulation. 1997;95(1):5-7.

32. Urbina EM, Srinivasan S, Kieltyka R, Tang R, Bond M, Chen W, et al. Correlates of carotid artery stiffness in young adults: the Bogalusa Heart Study. Atherosclerosis. 2004;176(1):157-64.

33. Schaeffner ES, Kurth T, Curhan GC, Glynn RJ, Rexrode KM, Baigent C, et al. Cholesterol and the risk of renal dysfunction in apparently healthy men. J Am Soc Nephrol. 2003;14(8):2084-91.

34. Li N, Fu J, Koonen DP, Kuivenhoven JA, Snieder H, Hofker MH. Are hypertriglyceridemia and low $\mathrm{HDL}$ causal factors in the development of insulin resistance? Atherosclerosis. 2014;233(1):130-8.

35. Hosseinpanah F, Barzin M, Eskandary PS, Mirmiran P, Azizi F. Trends of obesity and abdominal obesity in Tehranian adults: a cohort study. BMC Public Health. 2009;9(1):426.

36. Kheirandish M, Asgari S, Lotfaliany M, Bozorgmanesh M, Saadat N, Tohidi $M$, et al. Secular trends in serum lipid levels of a Middle Eastern adult population; 10 years follow up in Tehran lipid and glucose study. Lipids Health Dis. 2014;13(1):20.

37. Arnett DK, McGovern PG, Jacobs DR Jr, Shahar E, Duval S, Blackburn $\mathrm{H}$, et al. Fifteen-year trends in cardiovascular risk factors (1980-1982 through 1995-1997) the Minnesota Heart Survey. Am J Epidemiol. 2002:156(10):929-35.

38. Palmer MK, Toth PP. Trends in lipids, obesity, metabolic syndrome, and diabetes mellitus in the United States: An NHANES analysis (2003-2004 to 2013-2014). Obesity. 2019;27(2):309-14.

39. Vartiainen E, Laatikainen T, Peltonen M, Juolevi A, Männistö S, Sundvall J et al. Thirty-five-year trends in cardiovascular risk factors in Finland. Int J Epidemiol. 2010;39(2):504-18.

40. Jayedi A, Rashidy-Pour A, Khorshidi M, Shab-Bidar S. Body mass index, abdominal adiposity, weight gain and risk of developing hypertension: a systematic review and dose-response meta-analysis of more than 2.3 million participants. Obesity reviews. 2018;19(5):654-67.

\section{Publisher's Note}

Springer Nature remains neutral with regard to jurisdictional claims in published maps and institutional affiliations.

Ready to submit your research? Choose BMC and benefit from:

- fast, convenient online submission

- thorough peer review by experienced researchers in your field

- rapid publication on acceptance

- support for research data, including large and complex data types

- gold Open Access which fosters wider collaboration and increased citations

- maximum visibility for your research: over 100M website views per year

At BMC, research is always in progress.

Learn more biomedcentral.com/submissions 